\title{
TTR
}

Traduction, terminologie, rédaction

\section{Nicole Nolette. Jouer la traduction : théâtre et hétérolinguisme au Canada francophone. Ottawa, Presses de l'Université d'Ottawa, 2015, 284 p.}

\section{Cédric Ploix}

Volume 29, numéro 1, 1er semestre 2016

URI : https://id.erudit.org/iderudit/1050718ar

DOI : https://doi.org/10.7202/1050718ar

Aller au sommaire du numéro

Éditeur(s)

Association canadienne de traductologie

ISSN

0835-8443 (imprimé)

1708-2188 (numérique)

Découvrir la revue

Citer ce compte rendu

Ploix, C. (2016). Compte rendu de [Nicole Nolette. Jouer la traduction : théâtre et hétérolinguisme au Canada francophone. Ottawa, Presses de l'Université

d'Ottawa, 2015, 284 p.] TTR, 29(1), 257-261. https://doi.org/10.7202/1050718ar d'utilisation que vous pouvez consulter en ligne.

https://apropos.erudit.org/fr/usagers/politique-dutilisation/ 


\section{References}

Lal, P. (1972). Transcreation: Two Essays. Kolkata, Writer's Workshop.

Meschonnic, Henri (1982). Critique du rythme. Anthropologie bistorique du langage. Lagrasse, Verdier.

St. André, James (2010). Thinking Through Translation with Metaphors. Manchester, St. Jerome Publishing.

Woodsworth, Judith (1988). "Metaphor and Theory: Describing the Translation Process.” In P. Nigel Chaffey, A. Fougner Rydning and S. Schult Ulriksen, eds. Translation Theory in Scandinavia: Proceedings from the Scandinavian Symposium on Translation Theory, Oslo 11-13 August 1988. Oslo, p. 281-299.

\section{Paul St-Pierre Université De MontréAL}

\section{Nicole Nolette. Jouer la traduction : théâtre et hétérolinguisme au Canada francophone. Ottawa, Presses de l'Université d'Ottawa, 2015, 284 p.}

Qu'on ne s'attende pas, à la lecture du titre, Jouer la traduction, à un ouvrage qui s'inscrit dans la perspective, devenue aujourd'hui quasiment désuète, d'une réflexion sur la distinction entre la traduction pour la page et la traduction pour la scène, et sur les possibilités et stratégies traductives visant à rendre le jeu théâtral performant sur la scène étrangère. Les références à la théorie générale de la relation texte-performance et de l'adaptation théâtrale n'apparaissent que brièvement dans le premier chapitre à travers le résumé de l'article "Théâtre et traduction : un aperçu du débat théorique» de Fabio Regattin, et ne constituent pas la base théorique centrale de l'ouvrage.

Ce n'est pas non plus une de ces études qui abordent la traduction comme une activité restrictive, subordonnée, invisible et placée sous l'égide de l'équivalence. Si Nicole Nolette fait une riche revue critique du discours sur l'intraduisibilité (p. 25-29), c'est pour s'en inscrire en faux : elle réhabilite la traduction d'une pleine positivité, en montrant, à travers l'analyse fine d'un corpus limité, que la traduction peut être une véritable source de création. De surcroît, dans les pièces choisies, la traduction est placée audevant de la scène, directement visible, faisant partie intégrante du spectacle. Grâce à l'effet métathéâtral qui résulte de cette présence, 
le statut du traducteur peut parfois se confondre avec celui du dramaturge ou du metteur en scène.

Enfin, ce n'est pas un ouvrage qui traite de la traduction comme une activité se situant nécessairement a posteriori : Nolette met autant l'accent sur les œuvres hétérolingues - comportant en elles-mêmes des stratégies d'accommodement destinées à un public unilingue, ou d'exclusion, engendrant un «rire de supériorité » chez le public bilingue - que sur la "retraduction» de ces œuvres. En cela, s'intégrant pleinement dans l'évolution du discours critique de la traduction au théâtre, Nolette se désolidarise de la définition traditionnelle de la traduction comme étant un mouvement linguistique et culturel du texte source au texte cible; la traduction nest plus un mouvement entre deux cultures, mais, elle-même, une expérience ontologiquement biculturelle.

L'auteure examine les différentes stratégies de mise en scène de l'hétérolinguisme théâtral, sa traduction et les mécanismes de sa réception suivant le profil linguistique des spectateurs. À travers l'analyse des textes, des mises en scène ainsi que des autres paratextes - critiques, surtitres, programmations de théâtre, etc. -, on a dans cet ouvrage une riche réflexion sur la traduction comme un élargissement infini des possibilités théâtrales : «La traduction ludique donne lieu tantôt à l'accumulation des jeux innocents, tantôt à une suppléance qui détraque les entités linguistiques et scéniques que l'on concevait comme déjà complètes» (p. 23).

Nolette se garde de ne prendre en compte que le plaisir et l'inventivité. En effet, la portée de l'ouvrage dépasse le simple pouvoir ludique de la traduction; le ludisme est chez l'auteure empreint de lucidité. Dans cette riche et convaincante étude sur le théâtre de l'exiguïté franco-canadienne des trois dernières décennies, l'auteure trace un panorama des principales institutions théâtrales francophones au Canada, et étudie au cas par cas le pouvoir de la traduction sur la mobilité des pièces hétérolingues et le processus de légitimation de celles-ci dans les métropoles et les institutions théâtrales au fonctionnement surtout unilingue. Mais l'auteure montre que loin de se cantonner à une résistance d'un communautarisme linguistique et d'une protection identitaire contre les hégémonies culturelles, la réinscription des pièces franco-canadiennes dans un nouvel espace linguistique est souvent plus un jeu qu'un enjeu : la tendance serait, du moins 
pour plusieurs pièces, d'entrelacer l'identitaire et l'universalisme post-identitaire.

Le premier chapitre pose les concepts et une importante revue de littérature, les chapitres suivants détaillent des études de cas, regroupées par zones géographiques, de l'ouest de l'Acadie en passant par l'Ontario francophone. Si cette structure traditionnelle a les vertus de la clarté et de l'équilibre, la rareté des exemples des pièces du corpus dans le premier chapitre a quelques fois le défaut de perdre le lecteur : le rapport entre jeu et traduction en général, par exemple, semble un peu forcé. Cependant, la dimension essentiellement théorique, la densité conceptuelle et la grande érudition du premier chapitre ne plongent pas pour autant le discours dans l'aridité : on apprécie la clarté du style et une structuration très détaillée. Si le premier chapitre est remarquablement instruit, on aurait aimé, en revanche, une distinction plus nette entre les termes «bilingue», «hétérolingue», "plurilingue», «diglossique», qui sont quelques fois utilisés de manière interchangeable dans l'ouvrage. Remarquons que Nolette n’emploie pas le terme "hétéroglossie», notamment utilisé par Marvin Carlson tout au long de son ouvrage Speaking in Tongues : Languages at Play in the Theatre (2006).

Les chapitres subséquents peuvent quasiment se lire de manière indépendante, suivant l'intérêt du lecteur pour telle ou telle zone géographique. En effet, les liens entre les différents chapitres et les remarques comparatives entre les différents exemples sont assez rares : la vue d'ensemble du théâtre hétérolingue francophone ne vient que dans la conclusion. Le lecteur n'a néanmoins pas besoin d'être incité à lire l'ensemble : il le fera très certainement de son propre chef, et avec le sourire aux lèvres, grâce à la dimension ludique des extraits du corpus, qui viennent ponctuer les analyses. Sur l'aspect purement formel, on regrette que l'auteure ait fait le choix de n'inscrire en index que les noms des institutions théâtrales, auteurs et metteurs en scène, et non les critiques, universitaires et autres références diverses : la maigreur de l'index ne reflète pas l'importante érudition de l'ouvrage et la longue bibliographie.

Si Nolette fait preuve d'une grande minutie dans l'étude dramaturgique de la traduction ludique sur scène, elle ne se lance pas dans de semblables développements sur la dimension purement esthétique de l'hétérolinguisme scénique, en dépit 
de ce que laisse entendre Doris Sommer, citée à la page 24 : «[t]he games of inclusion and exclusion (what linguists call "gatekeeping») produce artful effects that depend on a range of possible receptions" (2004, p. 64). Nolette fait à juste titre quelques remarques sur la musicalité de l'écriture poétique hétérolingue de l'adaptation théâtrale de L'homme invisiblel The Invisible Man (p.159-167), mais on aurait apprécié, ici et ailleurs, un travail plus poussé sur le rythme et la musicalité textuels, la polyphonie linguistique et les jeux vocaux lors des représentations. Certains objets d'étude de l'ouvrage sont déjà développés par Louise Ladouceur dans l'article «Exploring a Bilingual Aesthetics through Translation in Performance", où figurent quelques paragraphes sur le bilinguisme sur scène et le rôle des surtitres dans Je mén vais à Régina de Roger Auger et Sex, Lies et les Franco-Manitobains de Marx Prescott. Si Nolette va, évidemment bien plus loin dans l'analyse de ces pièces, on est cependant en droit d'être surpris de ne pas voir cet article figurer dans la bibliographie.

Cette étude est l'aboutissement d'un intérêt de plus en plus marqué pour le théâtre hétérolingue dans le discours général sur la traduction au théâtre : Cristina Marinetti, à la fin de son article "Transnational, Multilingual, and Post-dramatic», appelle à réfléchir sur le rôle et la position de l'interprète sur la scène. Dans le corpus choisi, la figure de l'interprète y tient justement une place importante.

Étude évidemment fondamentale au Canada, elle pourra aussi être utile à ceux qui s'intéressent au contexte de production théâtral aux États-Unis et en Amérique du Sud, aujourd'hui fortement multiculturel; pour citer un ouvrage critique absent de la bibliographie : «[T]ranslation in the Americas is less something that happens between separate and distinct cultures and more something that is constitutive of those cultures» (Gentzler, 2008, p. 5). Nolette s'intègre effectivement dans un discours émergeant : mentionnons, par exemple, les entreprises de reterritorialisation des pièces classiques dans un espace transnational, comme le montre William Worthen dans Shakespeare and the Force of Modern Performance (p. 117) ou les expériences multilingues de certains groupes de théâtre européens, notamment la Tara Arts Company à Londres. Si le ludisme n'est pas forcément leur principale motivation, certains thèmes et stratégies, comme la bivalence 
stratégique, l'accommodement et résistance, remarquablement conceptualisés dans Nolette, se retrouvent dans leurs pratiques. La lecture de ce livre pourra mettre en perspective ces pratiques avec de riches éclairages. Les discours et critiques sur l'identitaire sur scène, l'hétérolinguisme, l'alternance codique, l'exclusion et l'inclusion des spectateurs monolingues viendront certainement se multiplier dans le contexte de la mondialisation, des grands mouvements de population et du développement de la littérature postcoloniale.

\section{References}

Carlson, Marvin (2006). Speaking in Tongues: Languages at Play in the Theatre. Ann Arbour, University of Michigan Press.

Gentzler, Edwin (2008). Translation and Identity in the Americas: New Directions in Translation Theory. Londres et New York, Routledge.

Ladouceur, Louise (2013). «Exploring a Bilingual Aesthetics through Translation in Performance». In S. Bigliazzi, P. Kofler et P. Ambrosi, dir. Theatre Translation in Performance. Londres et New York, Routledge, p. 111-129.

Marinetti, Christina (2013). "Transnational, Multilingual, and Postdramatic: Rethinking the Location of Translation in Contemporary Theatre». In S. Bigliazzi, P. Kofler et P. Ambrosi, dir. Theatre Translation in Performance. New York, Routledge, p. 27-36.

Regattin, Fabio (2004). "Théâtre et traduction: un aperçu du débat théorique». L'annuaire théâtrale: revue québécoise d'études théâtrales, 36, p. 156-171.

Sommer, Doris (2004). Bilingual Aesthetics, a New Sentimental Education. Durham, Duke University Press.

Worthen, William (2003). Shakespeare and the Force of Modern Performance. Cambridge, Cambridge University Press.

\section{CÉdric Ploix UNIVERSITY OF OXFORD}

